\title{
Joint Scheduling and Precoding for Frame-Based Multigroup Multicasting in Satellite Communications
}

\author{
Ashok Bandi, Bhavani Shankar Mysore R, Symeon Chatzinotas, and Björn Ottersten \\ Interdisciplinary Centre for Security, Reliability and Trust (SnT), the University of Luxembourg, Luxembourg. \\ Email: \{ashok.bandi, bhavani.shankar, symeon.chatzinotas, bjorn.ottersten\}@uni.lu
}

\begin{abstract}
Recent satellite standards enforce the coding of multiple users' data in a frame. This transmission strategy mimics the well-known physical layer multigroup multicasting (MGMC). However, typical beam coverage with a large number of users and limited frame length lead to the scheduling of only a few users. Moreover, in emerging aggressive frequency reuse systems, scheduling is coupled with precoding. This is addressed in this work, through the joint design of scheduling and precoding for frame-based MGMC satellite systems. This aim is formulated as the maximization of the sum-rate under per beam power constraint and minimum SINR requirement of scheduled users. Further, a framework is proposed to transform the non-smooth SR objective with integer scheduling and nonconvex SINR constraints as a difference-of-convex problem that facilitates the joint update of scheduling and precoding. Therein, an efficient convex-concave procedure based algorithm is proposed. Finally, the gains (up to $50 \%$ ) obtained by the jointed design over state-of-the-art methods is shown through Monte-Carlo simulations.
\end{abstract}

Index Terms-Scheduling, precoding, Multigroup multicast

\section{INTRODUCTION}

To meet the demands of data-hungry services from an everincreasing devices, multibeam architecture with aggressive frequency reuse is being considered as a solution [1]. To mitigate the inter-beam interference inherent in such systems, linear precoding is adopted [2] and has found support in the digital video broadcasting for satellite standard DVB-S2X [3]. However, several practical constraints need to be addressed for realizing precoding in practice. Firstly, in the DVB-S2X frame the data of multiple users are encoded within a single codeword before being incorporated in frames. Thus, the frame-based precoding resembles frame-based physical layer multigroup multicast (MGMC) precoding [4]. Secondly, onboard limitation of sharing the power across beams requires the per beam power constraints (PBPC).

In this work, we address the frame-based MGMC precoding for forward link of a multibeam system where each beam equipped with a single transmit antenna covers a large number of users equipped with single antenna receivers. Due to practical constraints such as limited power and frame length, only a few users can be accommodated into a frame transmitted over a beam. Naturally, this leads to the scheduling of

This work is supported in part by Luxembourgish national fund FNR project PROSAT. users that maximizes the objective of interest. In this work, the considered objective of interest is the sum of minimum rates of a beam over all the beams (SR). Clearly, that rate and hence the user scheduling in a beam is a function of signal-to-interference plus noise ratio (SINR). Moreover, the interference to users of a beam depends on the precoding, which in turn, depends on the scheduled users (SU) in other beams. Thus, the maximization of the objective requires the joint design of scheduling and precoding across all the users in all the beams. In this context, the joint design of scheduling and precoding simply refer to as joint design.

\section{A. Related works}

The sum-rate maximization (SRM) for MGMC was initially addressed in [5] under the sum power constraint. Therein, a heuristic algorithm based on decoupling of precoding and power allocation is proposed. The authors in [6] proposed a heuristic user scheduling and extended the precoding framework in [5] to SRM under PBPC for frame-based MGMC. Noticing the complexity of the algorithms in [5] and [6], the authors in [7] propose a heuristic two-stage low-complexity solution where the precoder design for inter-beam is followed by the precoder design for intra-beam interference. User scheduling is not addressed [5], [7] and the proposed user scheduling in [6] is heuristic. The precoding solutions proposed in [5], [7] and [6] for SRM problem are heuristic without any guarantees on performance. Moreover, as mentioned previously, the SRM problem entails a joint design of scheduling and precoding. Hence, the decoupled approach adopted in [6] provides only a feasible solution and results in loss of performance compared to the joint design methods. To the best of our knowledge, joint design for MGMC systems is not addressed in the literature.

To this end, the contributions of this work are as follows:

- To capture the coupled nature of scheduling and precoding, a novel formulation of the SRM problem is proposed that embodies scheduling aspects of the design with the help of binary variables besides precoding. Unlike the formulations in [5], [6], the proposed formulation results a continuous precoding problem for given SUs.

- With the help of novel reformulations, non-convex nature of the problem arising from SINR terms is transformed 
as a difference-of-convex/concave (DC) functions. The binary constraint is handled with appropriate relaxation and penalization. These reformulations render the joint design as a DC problem, a fact hitherto not considered.

- Within the framework of the convex-concave procedure (CCP), an iterative algorithm is proposed to solve the resulting DC problem wherein each iterate a convex problem is solved. Convergence to a stationary point is inherently guaranteed and performance is enhanced.

The sequel is organized as follows. Section II presents MGMC in satellite systems and the SRM problem. Section III presents the DC formulation of the problem; details of the CCP based algorithm are described in Section IV. Section V presents simulations and Section VI concludes the work.

Notation: Lower or upper case letters represent scalars, lower case boldface letters represent vectors, and upper case boldface letters represent matrices. $\|\cdot\|$ represents the Euclidean norm, $|\cdot|$ represents the cardinality of a set or the magnitude of a scalar depending on the argument, $(\cdot)^{H}$ represents Hermitian transpose and $\left(\begin{array}{l}a \\ b\end{array}\right)$ represents $a$ choose $b$

\section{MGMC SCENARIO AND PROBLEM FORMULATION}

\section{A. MGMC Scenario}

We consider the forward link of a geostationary satellite having multibeam capabilities and offering broadband services. Considering the evolving trends in satellite systems, full frequency reuse is considered for the multiple beams; this results in multi-user interference, whose mitigation is the key aspect of the paper. The system is assumed to support the DVB-S2x Physical layer based on superframing [3] to support the synchronized frames of the same length and the required CSI pilots as necessitated by precoding [6]. Further, the users are assumed to possess a single antenna with processing power to demodulate one data stream. Furthermore, the feeder link is assumed ideal and the satellite is operated in the linear mode. Finally, the service needed for much larger number of users compared to the number of beams is provided by scheduling different users in frames transmitted over time.

We consider a MGMC transmission in the aforementioned multibeam scenario comprising $N$ beams formed by $N$ transmit antennas. The beam $i$ serves a total of $U_{i}$ users and the total number of users in all beams is assumed greater than number of beams (also antennas) i.e., $\sum_{i=1}^{N} U_{i}>N$. In a given time slot, exactly say, $K_{i} \leq U_{i}$, users are served by multiplexing the users' data in a codeword designed for the transmission to users in beam $i$; this is referred to as frame-based multicasting [6]. The generic case of $K_{i} \geq 1$ arises from the need to use spectrally efficient long codewords while avoiding transmission inefficiency due to concatenating a user's data with sizable dummy bits in the codeword [6]. Further, due to the use of full frequency reuse, a multicast group of $K_{i}$ users in beam $i$ is interfered by $K_{j}, j \neq i$, users from other co-channel beams leading to the MGMC scenario. Since $K_{i} \leq U_{i}$, this naturally leads to the selection of $K_{i}$ of users out of $U_{i}$ which is referred to as user scheduling in this paper (which is also referred to equivalently as user selection or admission control in the literature).

\section{B. Performance metric and problem formulation}

In this work, we consider the sum of minimum rates achieved by each of the different multicast groups as the performance metric. This metric considers the minimum rate of SUs in each beam and summation is across the beams and will be simply referred to as a sum-rate henceforth (also in the literature in the context of MGMC [6]). In the sequel, we focus on maximization of this sum-rate subject to constraints on the number of SUs per beam, minimum SINR (MSINR) or equivalently minimum rate of SUs per beam and the consumed power per beam; this problem is compactly referred to $S R$ in the rest of paper.

Towards formulating the $\mathrm{SR}$, let $\mathbf{w}_{i} \in \mathbb{C}^{N \times 1}, P_{i}>0$ and $\epsilon_{i}>0$ be the precoding vector, maximum allowed per beam transmit power and MSINR (i.e., QoS) requirement of beam $i$ respectively. Noise at each user is characterized to be as additive white Gaussian with zero mean and variance $\sigma^{2}$. Let $\mathbf{h}_{i j} \in \mathbb{C}^{N \times 1}$, and $\gamma_{i j}=\frac{\left|\mathbf{h}_{i j}^{H} \mathbf{w}_{i}\right|^{2}}{\sum_{l \neq i}\left|\mathbf{h}_{i j}^{H} \mathbf{w}_{l}\right|^{2}+\sigma^{2}}$ be the downlink channel and SINR of user $j$ belonging to beam $i$ respectively. Let $\mathcal{S}_{i}$ be any subset of $\left\{1, \ldots, U_{i}\right\}$ with cardinality equal to $K_{i}$ and $\mathcal{T}_{i}$ be the dictionary of all sets of type $\mathcal{S}_{i}$. Clearly, the number of sets in $\mathcal{T}_{i}$ is $\left(\begin{array}{c}U_{i} \\ K_{i}\end{array}\right)$ for $i \in\{1, \ldots, N\}$. With the notations defined, the SR is formulated as,

$$
\begin{aligned}
& \mathcal{P}_{1}: \quad \max _{\left\{\mathbf{w}_{i}, \mathcal{S}_{i} \in \mathcal{T}_{i}\right\}_{i=1}^{N}} \sum_{i=1}^{N} \log \left(1+\Omega_{i}\right) \\
& \text { s.t. } C_{1}: \Omega_{i}=\min _{j \in \mathcal{S}_{i}} \gamma_{i j}, i \in\{1, \ldots, N\}, \\
& \quad C_{2}: \gamma_{i j} \geq \epsilon_{i}, i \in\{1, \ldots, N\}, j \in \mathcal{S}_{i}, \\
& \quad C_{3}: \sum_{j=1}^{N}\left|\mathbf{w}_{i j}\right|^{2} \leq P_{i}, i \in\{1, \ldots, N\} .
\end{aligned}
$$

Remarks:

- $\mathcal{S}_{i}$ contains the set of SUs in beam $i$, hence the constraint $C_{1}$ in $\mathcal{P}_{1}$ represents the MSINR of the SUs.

- Constraint $C_{2}$ in $\mathcal{P}_{1}$ enforces the SUs in each beam to satisfy the corresponding MSINR requirement associated with the beam. This enables the flexibility to support different rates on different beams.

- Constraint $C_{3}$ is the PBPC. This follows from the use of a separate transponder for each beam due to co-channel assumption [8].

- The objective in $\mathcal{P}_{1}$ denotes the sum-rate.

Notice that the problem $\mathcal{P}_{1}$ is coupled in two levels:

- Design of scheduler and precoder in a beam entails their joint design as the scheduling of a user depends on the precoder and the precoder design depends on the user.

- Design of scheduling and precoding in a beam requires joint design across all the beams as the precoder of a beam (which in turn depends on scheduled user) contributes to the interference of other beams. 
Hence, the optimal solution to problem $\mathcal{P}_{1}$ entails the joint design of scheduling and precoding where the scheduling is performed across all the users in all beams (multicast groups) and precoding is performed across all beams.

The problem $\mathcal{P}_{1}$ is combinatorial due to the selection of sets of users and also non-convex due to constraint $C_{1}$ and $C_{2}$. Hence, obtaining the optimal solution to $\mathcal{P}_{1}$ requires the exhaustive search algorithms whose complexity grows exponentially with increase in problem dimension (e.g., dictionary for each beam has dimensions $\left(\begin{array}{c}U_{i} \\ K_{i}\end{array}\right)$ and their joint design is entailed). Therefore, in the next section, we focus on transforming the problem $\mathcal{P}_{1}$ into a continuous problem and exploiting the hidden DC structure in the non-convexity.

\section{DC FORMULATION: A TRACTABLE APPROACH}

Towards formulating the problem $\mathcal{P}_{1}$ without the set notions, let $\eta_{i j} \in\{0,1\}$ be the binary variable associated with user $j$ in beam $i$, where $\eta_{i j}=1$ when the corresponding user is scheduled and zero otherwise. With the help $\eta_{i j}$ s, the problem $\mathcal{P}_{1}$ is reformulated as,

$$
\begin{aligned}
\mathcal{P}_{2}: & \max _{\mathbf{W}, \boldsymbol{\eta}, \boldsymbol{\Omega}} \sum_{i=1}^{N} \log \left(1+\Omega_{i}\right) \\
\text { s.t. } & C_{1}: \eta_{i j} \in\{0,1\}, \forall i, \forall j, \\
& C_{2}: \gamma_{i j} \geq \eta_{i j} \Omega_{i}, \forall i, \forall j, \\
& C_{3}: \Omega_{i} \geq \epsilon_{i}, \forall i, \\
& C_{4}: \sum_{j=1}^{U_{i}} \eta_{i j}=K_{i}, \forall i, \\
& C_{5}: \sum_{j=1}^{N}\left|\mathbf{w}_{i j}\right|^{2} \leq P_{i}, \forall i,
\end{aligned}
$$

where $\forall i$ refers to $i \in\{1, \ldots, N\}, \forall j$ refers to $j \in$ $\left\{1, \ldots, U_{i}\right\}, \mathbf{W}=\left[\mathbf{w}_{1}, \ldots, \mathbf{w}_{N}\right], \boldsymbol{\eta}_{i}=\left[\eta_{i 1}, \ldots, \eta_{i U_{i}}\right]^{T}$, for $i \in\{1, \ldots, N\}, \boldsymbol{\eta}=\left[\boldsymbol{\eta}_{1}, \ldots, \boldsymbol{\eta}_{N}\right]$ and $\boldsymbol{\Omega}=\left[\Omega_{1}, \ldots, \Omega_{N}\right]$. Remarks:

- When $\eta_{i j}=0$, the constraint $C_{2}$ imposes a trivial lower bound on SINR of the unscheduled user $j$ in beam $i$ i.e., $\gamma_{i j} \geq 0$. When $\eta_{i j}=1$, constraint $C_{2}$ leads to $\gamma_{i j} \geq \Omega_{i}$.

- Letting $U_{i}^{S}$ to be the set of SUs in beam $i$, the constraint $C_{2}$ ensures $\Omega_{i} \geq \min _{j \in U_{i}^{S}} \gamma_{i j}$. Hence, maximization of the objective in the problem $\mathcal{P}_{2}$ equivalently maximizes the sum-rate in $\mathcal{P}_{1}$.

- Constraint $C_{3}$ is the MSINR requirement of users.

- Constraint $C_{4}$ imposes a limit on the number of SUs in each beam; this ensures each user gets sufficient share of the physical layer frame.

Novelty of $\mathcal{P}_{2}$ : The proposed reformulation transforms non-smooth non-tractable joint design problem $\mathcal{P}_{1}$ into a tractable problem given in $\mathcal{P}_{2}$. Moreover, this reformulation is crucial for the reformulations that are proposed in sequel to transform $\mathcal{P}_{1}$ to a smooth DC problem. The novelty mainly lies in the reformulation of $C_{1}$ in $\mathcal{P}_{1}$ to $C_{2}$ in $\mathcal{P}_{2}$. To the best of our knowledge, the formulation given in $\mathcal{P}_{2}$ is the first of its kind that captures both scheduling and precoding.
The problem $\mathcal{P}_{2}$ is non-smooth and combinatorial due to constraint $C_{1}$; further the constraint $C_{2}$ is non-convex. Towards uncovering structure in the non-convexity, let $\beta_{i j}$ be the slack variable for lower bounding the SINR of user $j$ and beam $i$. A reformulation of $\mathcal{P}_{2}$ with the help of $\beta_{i j} s$ is,

$$
\begin{aligned}
\mathcal{P}_{3}: & \max _{\mathbf{W}, \boldsymbol{\eta}, \boldsymbol{\Omega}, \boldsymbol{\beta}} \sum_{i=1}^{N} \log \left(1+\Omega_{i}\right) \\
\text { s.t. } & C_{1}, C_{3}, C_{4}, C_{5} \text { in }(2) \\
& C_{2}: \gamma_{i j} \geq \beta_{i j}, \forall i, \forall j, \\
& C_{6}: \beta_{i j} \geq \eta_{i j} \Omega_{i}, \forall i,
\end{aligned}
$$

where $\boldsymbol{\beta}=\left[\boldsymbol{\beta}_{i}, \ldots, \boldsymbol{\beta}_{N}\right]$ and $\boldsymbol{\beta}_{i}=\left[\beta_{i 1}, \ldots, \beta_{i U_{i}}\right]^{T}$. Following [9], a DC reformulation of constraint $C_{2}$ in $\mathcal{P}_{2}$ is,

$$
C_{2} \Rightarrow 1+\gamma_{i j} \geq 1+\beta_{i j} \Rightarrow \mathcal{I}_{i j}(\mathbf{W})-\mathcal{J}_{i j}\left(\mathbf{W}, \beta_{i j}\right) \leq 0,
$$

where $\mathcal{I}_{i j}(\mathbf{W})=\sigma^{2}+\sum_{l \neq i}^{N}\left|\mathbf{h}_{i j}^{H} \mathbf{w}_{l}\right|^{2}$ and $\mathcal{J}_{i j}\left(\mathbf{W}, \beta_{i j}\right)=$ $\frac{\sigma^{2}+\sum_{l=1}^{N}\left|\mathbf{h}_{i j}^{H} \mathbf{w}_{l}\right|^{2}}{1+\beta_{i j}}$. Notice that $\mathcal{I}_{i j}(\mathbf{W})$ is convex and $\mathcal{J}_{i j}\left(\mathbf{W}, \beta_{i j}\right)$ is also jointly convex in $\mathbf{W}$ and $\beta_{i j}$. Hence, by the equivalent reformulation given in (4), the constraint $C_{2}$ in $\mathcal{P}_{3}$ is a DC constraint. Further, a DC form of $C_{6}$ is,

$$
C_{6}: 4 \beta_{i j}+\left(\eta_{i j}-\Omega_{i}\right)^{2} \geq\left(\eta_{i j}+\Omega_{i}\right)^{2} .
$$

With the reformulations given in (4) and (5), and ignoring the combinatorial constraint $C_{1}$ for the moment, the problem $\mathcal{P}_{3}$ leads to the maximization of concave objective subject to difference-of-convex, convex and linear constraints; this is a DC problem [10] and can be solved efficiently by CCP [10].

As the final step, the combinatorial constraint $C_{1}$ is addressed by relaxing $\eta_{i j} \mathrm{~s}$ to a box constraint between 0 and 1 i.e., $0 \leq \eta_{i j} \leq 1$. This relaxation along with the aforementioned reformulations renders $\mathcal{P}_{3}$ as a DC programming problem. However, the resulting $\eta_{i j}$ s obtained with this relaxation might be non-binary. Hence, to ensure their binary nature, $\eta_{i j}$ is penalized with $\mathbb{P}\left(\eta_{i j}\right)$. Letting $\lambda$ to be the penalty parameter, the resulting penalized reformulation is,

$$
\begin{aligned}
\mathcal{P}_{4}: & \max _{\mathbf{W}, \boldsymbol{\eta}, \boldsymbol{\Omega}, \boldsymbol{\beta}} \sum_{i=1}^{N}\left(\log \left(1+\Omega_{i}\right)+\lambda \sum_{j=1}^{U_{i}} \mathbb{P}\left(\eta_{i j}\right)\right) \\
\text { s.t. } & C_{1}: 0 \leq \eta_{i j} \leq 1, \forall i, \forall j, \\
& C_{2}: \mathcal{I}_{i j}(\mathbf{W})-\mathcal{J}_{i j}\left(\mathbf{W}, \beta_{i j}\right) \leq 0, \forall i, \forall j \\
& C_{3}, C_{4}, C_{5} \text { in }(3) \\
& C_{6}: 4 \beta_{i j}+\left(\eta_{i j}-\Omega_{i}\right)^{2} \geq\left(\eta_{i j}+\Omega_{i}\right)^{2}, \forall i, \forall j
\end{aligned}
$$

It is easy to see that any choice of convex function $\mathbb{P}\left(\eta_{i j}\right)$ that promotes the binary solutions suffice to transform $\mathcal{P}_{4}$ as a DC problem of our interest. For example, the penalty functions proposed in [9] and [11] can be chosen as $\mathbb{P}\left(\eta_{i j}\right)$. The log-entropy based penalty function proposed in [9] i.e., $\mathbb{P}\left(\eta_{i}\right) \triangleq \eta_{i} \log \eta_{i}+\left(1-\eta_{i}\right) \log \left(1-\eta_{i}\right)$ is considered for this work. With this choice of $\mathbb{P}\left(\eta_{i}\right)$, the problem $\mathbb{P}\left(\eta_{i}\right)$ becomes a DC problem. In order to apply the CCP framework to the problem $\mathbb{P}\left(\eta_{i}\right)$, a feasible initial point (FIP) needs to supplied. 
However, the constraint $C_{4}$ in $\mathbb{P}\left(\eta_{i}\right)$ limits the choices of FIPs. For ease of finding the FIPs, the constraint $C_{2}$ is brought into the objective with another penalty parameter $\gamma>0$ as,

$$
\mathcal{P}_{5}: \max _{\mathbf{W}, \boldsymbol{\eta}, \boldsymbol{\Omega}, \boldsymbol{\beta}} \mathcal{F}(\boldsymbol{\Omega}, \boldsymbol{\eta}) \text { s.t. } C_{1}, C_{2}, C_{3}, C_{5}, C_{6} \text { in (6). }
$$

where $\mathcal{F}(\boldsymbol{\Omega}, \boldsymbol{\eta})=\sum_{i=1}^{N}\left(\log \left(1+\Omega_{i}\right)+\lambda \sum_{j=1}^{U_{i}} \mathbb{P}\left(\eta_{i j}\right)\right)$ $-\sum_{i=1}^{N} \gamma\left(\sum_{j=1}^{U_{i}} \eta_{i j}-K_{i}\right)$. Given the non-emptyness of the solution set, a solution of the problem $\mathcal{P}_{4}$ is always obtained by solving $\mathcal{P}_{5}$ with right choice of $\gamma$ (usually larger value).

\section{CCP BASEd JoInt DESIGN AlgorithM}

In this section, a CCP [10] based algorithm is proposed to solve the DC problem in (6). CCP is an efficient tool to find a stationary point of DC programming problems [12]. It is an iterative framework wherein the two steps of Convexification and Optimization are executed in each iteration. In the convexification step, the DC problem is approximated as a convex problem by linearizing the convex part of the objective and the concave part of the DC constraints by their first-order Taylor approximations. The convex problem obtained from convexification step provides a global lower bound for the original problem where the lower bound is tight at the previous iteration. The optimization step involves the maximization of the lower bound obtained from Convexification step.

\section{A. Joint Scheduling and Precoding (JSP) Algorithm}

The convexifcation and optimization steps of CCP framework, applied to the DC problem $\mathcal{P}_{5}$, are as follows,

- Convexification: Let $\left(\mathbf{W}^{k-1}, \boldsymbol{\eta}^{k-1}, \boldsymbol{\beta}^{k-1}, \boldsymbol{\Omega}^{k-1}\right)$ be the estimates of $(\mathbf{W}, \boldsymbol{\eta}, \boldsymbol{\beta}, \boldsymbol{\Omega})$ in iteration $k-1$ respectively. In iteration $k$, the convex part of the objective, $\left(\sum_{i=1}^{N} \lambda \mathbb{P}\left(\eta_{i}\right)\right)$ is replaced by

$$
\tilde{\mathbb{P}}\left(\eta_{i}\right) \triangleq \lambda\left(\mathbb{P}\left(\eta_{i}^{k-1}\right)+\left(\eta_{i}-\eta_{i}^{k-1}\right) \nabla \mathbb{P}\left(\eta_{i}^{k-1}\right)\right),
$$

the concave part of $C_{2}$ i.e., $\mathcal{J}_{i j}\left(\mathbf{W}, \beta_{i j}\right)$ is replaced by

$$
\begin{aligned}
& \tilde{\mathcal{J}}_{i j}\left(\mathbf{W}^{k-1}, \beta_{i j}^{k-1}\right) \triangleq-\mathcal{J}_{i j}\left(\mathbf{W}, \beta_{i j}\right)- \\
& \Re\left\{\operatorname{Tr}\left\{\nabla^{H} \mathcal{J}_{i}\left(\mathbf{W}^{k-1}, \beta_{i j}^{k-1}\right)\left[\begin{array}{c}
\mathbf{w}_{1}-\mathbf{w}_{1}^{k-1} \\
\vdots \\
\mathbf{w}_{N}-\mathbf{w}_{N}^{k-1} \\
\beta i j-\beta_{i j}^{k-1}
\end{array}\right]\right\},\right.
\end{aligned}
$$

and concave part of $C_{6}$ i.e., $\left(\eta_{i j}-\Omega_{i}\right)^{2}$ is replaced by

$$
\begin{aligned}
\mathcal{G}_{i j}\left(\eta_{i j}, \Omega_{i}\right) & \triangleq\left(\eta_{i j}^{k-1}-\Omega_{i}^{k-1}\right)^{2}+ \\
& {\left[\begin{array}{c}
2\left(\eta_{i j}^{k-1}-\Omega_{i}^{k-1}\right) \\
-2\left(\eta_{i j}^{k-1}-\Omega_{i}^{k-1}\right)
\end{array}\right]^{T}\left[\begin{array}{c}
\left(\eta_{i j}^{k-1}-\eta_{i j}\right) \\
\left(\Omega_{i}^{k-1}-\Omega_{i}\right)
\end{array}\right] . }
\end{aligned}
$$

- Optimization: Updated $\left(\mathbf{W}^{k+1}, \boldsymbol{\Omega}^{k+1}, \boldsymbol{\eta}^{k+1}, \boldsymbol{\beta}^{k+1}\right)$ is obtained by solving the following convex problem,

$$
\begin{aligned}
\mathcal{P}_{6}: & \max _{\mathbf{W}, \boldsymbol{\eta}, \boldsymbol{\Omega}, \boldsymbol{\beta}} \mathcal{F}(\boldsymbol{\Omega}, \boldsymbol{\eta}) \\
& C_{1}: 0 \leq \eta_{i j} \leq 1, \forall i, \forall j,
\end{aligned}
$$

$$
\begin{aligned}
& C_{2}: \mathcal{I}_{i j}(\mathbf{W})-\tilde{\mathcal{J}}_{i j}\left(\mathbf{W}^{k-1}, \beta_{i j}^{k-1}\right) \leq 0, \forall i, \forall j \\
& C_{3}: \Omega_{i} \geq \epsilon_{i}, \forall i, \\
& C_{4}: \sum_{j=1}^{N}\left|\mathbf{w}_{i j}\right|^{2} \leq P_{i}, \forall i, \\
& C_{5}: 4 \beta_{i j}+\mathcal{G}_{i j}\left(\eta_{i j}, \Omega_{i}\right) \geq\left(\eta_{i j}+\Omega_{i}\right)^{2}, \forall i, \forall j
\end{aligned}
$$

The problem in $\mathcal{P}_{6}$ optimizes the sum-rate over scheduling and precoding variables jointly. This joint scheduling and precoding (JSP) algorithm is based on CCP framework. It is well known that a FIP is sufficient for the CCP procedure to converge to a stationary point (kindly refer [13]). The QOS problem for fixed $\boldsymbol{\eta}$ can be solved using [14]. Let $\hat{\mathbf{W}}$ be the solution for fixed $\boldsymbol{\eta}=\delta$ where $0 \leq \delta \leq 1$ is constant. For $\delta \approx 0$, the corresponding QOS problem always become feasible ( kindly refer to [14]). So, a FIP of the problem $\mathcal{P}_{5}$ can be $(\hat{\mathbf{W}}, \hat{\boldsymbol{\eta}}, \hat{\boldsymbol{\beta}}, \hat{\boldsymbol{\Omega}})$ where $\hat{\boldsymbol{\beta}}$ is the SINRs obtained with $\hat{\mathbf{W}}, \hat{\boldsymbol{\eta}}=\delta$ and $\hat{\Omega}_{i}=\epsilon_{i}$ for $i=1, \ldots, N$.

\section{B. Complexity of JSP and its reduction}

Since JSP is a CCP based iterative algorithm, its complexity depends on complexity of the convex sub-problem $\mathcal{P}_{5}$. The convex problem $\mathcal{P}_{5}$ has $\left(N^{2}+2 \sum_{i=1}^{N} U_{i}+N\right)$ decision variables and $\left(2 \sum_{i=1}^{N} U_{i}+N\right)$ convex constraints and $\left(2 \sum_{i=1}^{N} U_{i}+2 N\right)$ linear constraints. Hence, the computational complexity of $\mathcal{P}_{\nabla}$ is $\mathcal{O}\left(\left(N^{2}+2 \sum_{i=1}^{N} U_{i}+N\right)^{3}\left(4 \sum_{i=1}^{N} U_{i}+3 N\right)\right)$

Commercial software such as CVX can solve the convex problem of type $\mathcal{P}_{5}$ efficiently. Besides the complexity per iteration, the overall complexity also depends on the convergence speed of the algorithm. Through simulations, we observe that the JSP converges typically in 20-30 iterations.

1) Pre-selection of Users for Large System Dimensions: The proposed JSP algorithm is computationally efficient for small to medium system dimensions. However, the complexity per iteration of $\mathcal{P}_{5}$ grows exponentially as the system dimension increases. Such a situation is inherent in satellite systems with increasing user base per beam. A case in point is the satellite system (for which we present numerical results in the next section) with $N=9$ and $\left\{U_{i}=100, K_{i}=2\right\}_{i=1}^{N}$; the number of users is much larger than what could be accommodated in a beam. Such scenarios inhibit the applicability of JSP or the obtained solution may become obsolete due to lengthier processing times. However, for special cases of large dimension systems with $\left\{U_{i} \gg K_{i}\right\}_{i=1}^{N}$, the proposed joint design algorithm can be still applied by adopting the following two step process:

- Pre-selection: In this step, a small subset of users, say $\zeta_{i}$, in beam $i$, for $i=1, \ldots, N$ is selected based on some scheduling scheme. This is step is referred to as pre-selection. Typically $\zeta_{i}$ is chosen as $K_{i} \leq \zeta_{i} \leq U_{i}$.

- Joint design for the pre-selected users: In this step, the proposed JSP algorithm is employed for the beams with 
TABLE I

SIMULATION PARAMETERS

\begin{tabular}{|l|l|}
\hline Frequency Band & $\mathrm{Ka}(20 \mathrm{GHz})$ \\
\hline User terminal clear sky temp, $T_{\mathrm{cs}}$ & $235.3 \mathrm{~K}$ \\
\hline User Link Bandwidth, $B_{u}$ & $500 \mathrm{MHz}$ \\
\hline Output BackOFF, OBO & $5 \mathrm{~dB}$ \\
\hline On board Power, $P_{\text {tot }}$ & $50 \mathrm{dBW}$ \\
hline Atmospheric fading & Rain attenuation [16] \\
\hline Roll-off factor, $\alpha$ & 0.20 \\
\hline User terminal antenna gain, $G_{R}$ & $41.7 \mathrm{dBi}$ \\
\hline Multibeam Antenna Gain, $G_{i j}$ & Ref: [16] \\
\hline
\end{tabular}

pre-selected users to jointly schedule $K_{i}$ users out of $\zeta_{i}$ and design corresponding precoders.

The proposed pre-selection based JSP with two step process is simply referred to PS-JSP. The values of $\left\{\zeta_{i}\right\}_{i=1}^{U}$ are chosen such that the complexity of proposed JSP algorithm is affordable. The proposed two step process typically results in loss of performance in comparison with JSP algorithm employed for original system dimension. This performance loss is typically a function of the scheduling scheme employed in pre-selection step and also on $\left\{K_{i}-\zeta_{i}\right\}_{i=1}^{N}$.

\section{Simulation Results}

In this section, the performance of JSP and PS-JSP are evaluated based on the system setup described in Section II and simulation model defined in [16]. We consider the Shannon rate for JSP and PS-JSP for its analytical appeal. However, in satellite systems, the throughput is defined by the modulation and coding (MODCOD) schemes used. In this context, while the optimization problem is solved for the Shannon rate, the resulting SINRs are used to compute the following metric,

$$
R_{\mathrm{avg}}=\frac{2 B_{u}}{1+\alpha} \frac{1}{N} \sum_{i=1}^{N} f_{\text {DVB-S2X }}\left(\min _{i \in \hat{\mathcal{S}}_{i}}\left\{\operatorname{SINR}_{i}\right\}\right) .
$$

This metric, in [Gbps/beam], represents average user throughput when using DVB-S2x framing; the relevant parameters are defined in Table I. The function $f_{\text {DVB-S2X }}$ in (9) maps the received MSINR of a beam to the highest MODCOD scheme (defined in [3]) that can be supported. In this work, a MSINR threshold $\epsilon_{i}$ of $-2.85 \mathrm{~dB}$ corresponding to minimum transmission and $\sigma^{2}$ is assumed to be $0 \mathrm{~dB}$ at all user terminals. Number of beams $N$ is fixed to be 9 for all results. Further, the results are averaged over 100 different channel realizations.

The solution proposed in [6] is considered as a benchmark (BM) for all the performance comparisons. As discussed previously, the joint design entails the design of scheduling and precoding across all the users in all the beams jointly. The BM algorithm proposed in [6] is based on the decoupled design of scheduling followed by precoding. Moreover, the scheduling and precoding algorithms proposed in [6] are heuristic methods without any guarantees on the nature of the solutions. On the contrary, the proposed JSP algorithm jointly designs the scheduling and precoding over all the users in all beams and PS-JSP jointly design scheduling and precoding for the given subset of users in all the beams;

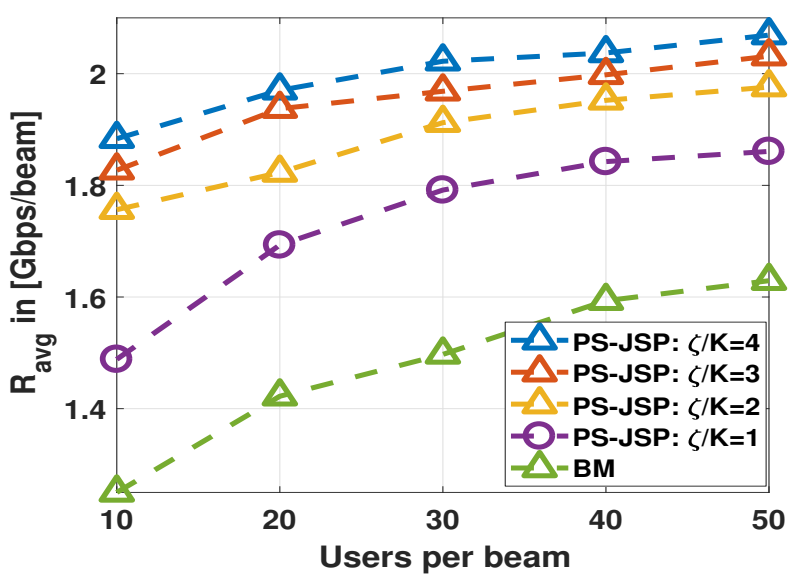

Fig. 1. Performance comparison of $R_{\text {avg }}$ (in Gbps/beam) versus $U_{i}$ for $N=$ $9,\left\{K_{i}=2, P_{i}=11.11 \text { Watts }\right\}_{i=1}^{N}$.

further, the optimization algorithm is based on a CCP which inherently provides qualifications on the solution. Hence, the gains obtained by JSP or PS-JSP is attributed to two factors: (i) improved precoder design per-se even for a given user set, (ii) jointly optimizing scheduling and precoding. Through the following numerical results, the gains in different scenarios are quantified. These scenarios are reflective of the typical operational aspects encountered in satellite communications.

\section{A. $R_{a v g}$ as a function of total users per beam}

It is quite essential in satellite communications to achieve higher spectral efficiencies with limited power. In figure 1, $R_{\text {avg }}$ is illustrated as a function of total users per beam for a limited power of 100 Watts. The number of users per beam $\left\{U_{i}=\Delta\right\}_{i=1}^{N}$ is varied from 10 to 50 insteps of 10 , and users per frame $\left\{K_{i}\right\}_{i=1}^{N}$ is fixed to be 2 . Recalling the PSJSP, the joint design is carried out on a pre-selected user pool containing $\zeta_{i}$ users in the $i$ th beam; here it is further assumed that $\zeta=\zeta_{i}, \forall i$. Figure 1 further illustrates the impact of choosing different $\zeta$. The scheduling algorithm proposed in [6] is employed to pre-select $\zeta$ users from $U_{i}$ in the preselection step of PS-JSP. Following are the gains obtained by JSP and PS-JSP over BM for this scenario:

- Precoding gain: For $\left\{\zeta_{i}=K_{i}=2\right\}_{i=1}^{N}$, the scheduling algorithms employed in PS-JSP and BM are the same leading to same set of SUs. In other words, the second step of PS-JSP (i.e., JSP) essentially designs only the precoder as $\left\{\zeta=K_{i}\right\}_{i=1}^{N}$. So, the gains obtained by PS-JSP over BM for $\zeta_{i}=K_{i}$, which amounts to approximately $12 \%$, is solely attributed to efficiency in the precoding of JSP which is introduced before as precoding gains.

- Joint optimization gain: For $\left\{\zeta_{i}>K_{i}\right\}_{i=1}^{N}$, the gains of PS-JSP over BM is due to both scheduling and precoding. In other words, the JSP step of PS-JSP schedules the users that contribute less interference to other beams and also users that consume less power to meet the MSINR requirements. The gains obtained by PS-JSP for $\left\{\frac{\zeta_{i}}{K_{i}}=4\right\}_{i=1}^{N}$ amounts to approximately $50 \%$ for 
$\left\{U_{i}\right\}_{i=1}^{N}=10$ (and $20 \%$ for $\left\{U_{i}\right\}_{i=1}^{N}=50$ ) is referred to as joint optimization gains.

Multiuser Diversity: Due to increased diversity in selecting users, (MUD: multiuser user diversity), the performance of PS-JSP improves as $\left\{\zeta_{i}\right\}_{i=1}^{N}$ increases. For example, when $\left\{U_{i}=10\right\}_{i=1}^{N}$, the gain for $\left\{\zeta_{i}=4 K_{i}\right\}_{i=1}^{N}$ is larger than that for $\left\{\zeta_{i}<4 K_{i}\right\}_{i=1}^{N}$ as the former case benefits from higher MUD. In other words, as $\left\{\zeta_{i}\right\}_{i=1}^{N}$ increases, the probability of finding the orthogonal users with good channel gains across beams increases and parallel users within a beam. This is shown in figure 1 . However, the gains diminish as $\zeta$ increases; additional users included due to increase in $\zeta$ tend to be less orthogonal with users in other beams and possess low correlation with users of the same beam. Similarly, as users per beam increases, the aforementioned probability increases, Hence, the performance of BM and PS-JSP improves as the number of users per beam increases initially, but these gains per-se diminish with further increase in users per beam.

\section{B. $R_{\text {avg }}$ as a function of users per frame}

The throughput of PS-JSP against BM is illustrated in figure 2 as a function of number of users per frame i.e., $\left\{K_{i}\right\}_{i=1}^{N}$. It is easy to see that due to systematic joint design, PS-JSP outperforms the BM. Similar to figure 1, for $\left\{\zeta_{i}=K_{i}\right\}_{i=1}^{N}$ the gains obtained by PS-JSP is solely attributed to the precoding gains and the gains for $\left\{\zeta_{i}>K_{i}\right\}_{i=1}^{N}$ are attributed the joint scheduling and precoding gains. The performance in a beam ( or frame) is dependent on the MSINR of the frame. Notice, scheduling in BM and pre-selection in PS-JSP are based on orthogonality of users across beams and co-channel nature of users within the beam. Hence, the newly SUs in a frame compared to the already SUs are less orthogonal to SUs in other beams. Hence, newly SUs increases interference. Further, due to the nature of adopted scheduling newly SUs have less channel gains. Hence, the increased interference with more SUs and decreased channel gains of the newly SUs lead to overall lower MSINR of a frame. As a result, $R_{\text {avg }}$ decreases as $\left\{K_{i}\right\}_{i=1}^{N}$ increases which is shown in figure 2 .

\section{CONClusions}

In this paper, the joint scheduling and precoding problem was considered for a MGMC scenario in frame-based satellite systems. Unlike the existing works, the joint design problem is formulated that facilitates the update of scheduling and precoding jointly. Noticing the problem to be MINLP, an efficient framework is developed that transform it as a DC problem. Finally, an efficient low-complexity CCP based iterative algorithm is proposed. Through Monte-Carlo simulations, the superiority in performance of the proposed algorithm over the state-of-the-art methods is illustrated.

\section{REFERENCES}

[1] M. . Vzquez, M. R. B. Shankar, C. I. Kourogiorgas, P. Arapoglou, V. Icolari, S. Chatzinotas, A. D. Panagopoulos, and A. I. Prez-Neira, "Precoding, scheduling, and link adaptation in mobile interactive multibeam satellite systems," IEEE J. Sel. Areas Commun., vol. 36, no. 5, pp. 971-980, May 2018.

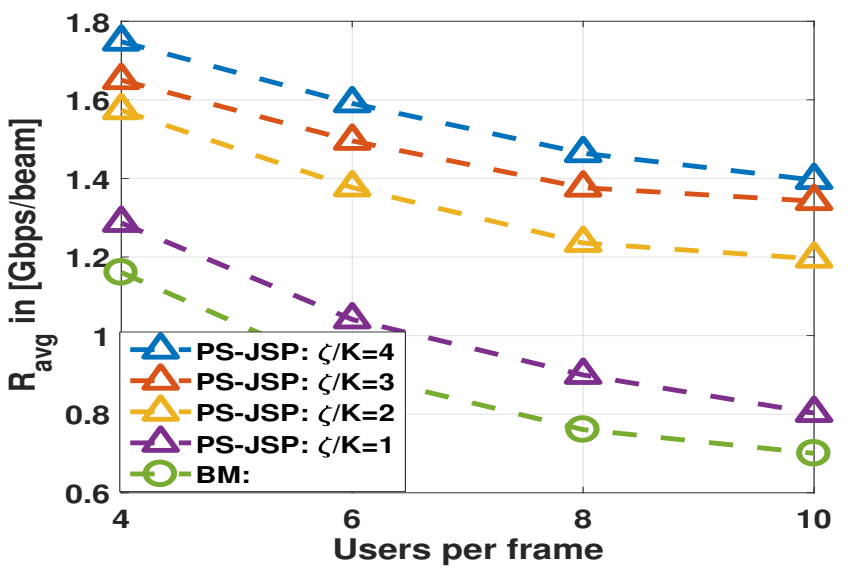

Fig. 2. Performance comparison of $R_{\text {avg }}$ in Gbps/beam versus $U_{i}$ for $N=9$, $\left\{U_{i}=100, P_{i}=11.11 \text { Watts }\right\}_{i=1}^{N}$.

[2] M. A. Vazquez, A. Perez-Neira, D. Christopoulos, S. Chatzinotas, B. Ottersten, P.-D. Arapoglou, A. Ginesi, and G. Tarocco, "Precoding in multibeam satellite communications: Present and future challenges," Wireless Commun., vol. 23, no. 6, pp. 88-95, Dec. 2016. [Online] Available: https://doi.org/10.1109/MWC.2016.1500047WC

[3] Digital Video Broadcasting (DVB); Second Generation Framing Structure, Channel Coding and Modulation Systems for Broadcasting, Interactive Services, News Gathering and Other Broad-Band Satellite Applications. Part 2: DVB-S2 Extensions (DVB-S2X), European Broadcasting Union (EBU),

[4] E. Lagunas, S. Andrenacci, S. Chatzinotas, and B. Ottersten, "Crosslayer forward packet scheduling for emerging precoded broadband multibeam satellite system," in 2018 9th ASMS/ 15th SPSC), Sep. 2018, pp. $1-8$.

[5] M. Kaliszan, E. Pollakis, and S. Staczak, "Multigroup multicast with application-layer coding: Beamforming for maximum weighted sum rate," in 2012 IEEE WCNC conference, April 2012, pp. 2270-2275.

[6] D. Christopoulos, S. Chatzinotas, and B. Ottersten, "Multicast multigroup precoding and user scheduling for frame-based satellite communications," IEEE Trans. Wireless Commun., vol. 14, no. 9, pp. 46954707, Sep. 2015.

[7] V. Joroughi, M. . Vzquez, and A. I. Prez-Neira, "Generalized multicast multibeam precoding for satellite communications," IEEE Trans. Wireless Commun., vol. 16, no. 2, pp. 952-966, Feb 2017.

[8] D. Christopoulos, S. Chatzinotas, and B. Ottersten, "Frame based precoding in satellite communications: A multicast approach," in 2014 7th ASMS Conferencel 13th SPSC workshop, Sep. 2014, pp. 293-299.

[9] A. Bandi, B. Shankar Mysore R, S. Chatzinotas, and B. Ottersten, "A Joint Solution for Scheduling and Precoding in Multiuser MISO Downlink Channels," arXiv e-prints, p. arXiv:1902.04415, Feb 2019.

[10] A. L. Yuille and A. Rangarajan, "The concave-convex procedure (CCCP)," in NIPS, 2001.

[11] A. Bandi and C. R. Murthy, "Structured sparse recovery algorithms for data decoding in media based modulation," in 2017 IEEE International Conference on Communications (ICC), May 2017, pp. 1-6.

[12] T. Lipp and S. Boyd, Variations and extension of the convex-concave procedure, http://stanford.edu/ boyd/papers/cvx_ccv.html, 2016.

[13] B. K. Sriperumbudur and G. R. G. Lanckriet, "On the convergence of the concave-convex procedure," in Neural Inf. Proc. Syst., Feb. 2009, pp. $1-9$.

[14] M. Bengtsson and B. Ottersten, "Optimal and suboptimal transmit beamforming," in Handbook of Antennas in Wireless Communications. CRC Press, 2001, pp. 18-1-18-33, qC 20111107.

[15] P. Gahinet, A. Nemirovski, A. J. Laub, and M. Chilali, LMI Control Toolbox Users Guide. USA: MathWorks,, 1995.

[16] Call of Order 2-Task 1: Fair comparison and combination of advanced interference mitigation techniques. 